\title{
A low dose of nicotine is sufficient to produce nicotine withdrawal in mice
}

\author{
Besson Morgane ${ }^{1,2}$, Suarez Sandra ${ }^{1}$, Changeux Jean-Pierre ${ }^{1}$, Granon Sylvie $^{1,3^{\star}}$ \\ ${ }^{1}$ Département de Neuroscience, Institut Pasteur, Paris, France; sylvie.granon@u-psud.fr \\ ${ }^{2}$ Department of Experiment Psychology, Downing Site, Cambridge, UK \\ ${ }^{3}$ Centre de Neuroscience Paris Sud, Université d’Orsay, Orsay, France
}

Received 12 October 2009; revised 12 November 2009; accepted 13 November 2009.

\begin{abstract}
The objective of our study was to investigate whether the chronic administration of a low dose of nicotine can be followed by a withdrawal syndrome at cessation of nicotine delivery. Previous studies showed various results, depending in the doses of nicotine, species, ways of administration and behavioural paradigms, but all emphasized a withdrawal effect on some or all of the following spontaneous behaviours: grooming, rearing, body shake or tremor, body scratching, abdominal constriction, jumping. However, it is not clear which behaviour is exactly altered, as a global behavioural index is most frequently used. This is not clear either if anxiety modulates the behavioral expression of withdrawal or which factors contribute to its locomotors effect, if any. To distant-angle these processes, we scored each of these behaviours individually before nicotine exposure, during continuous nicotine delivery and at cessation of nicotine delivery after precipitated withdrawal by mecamylamine injection. We also measured locomotor activity and anxiety levels in the same animals. We used a low dose of nicotine $(2.4 \mathrm{mg} / \mathrm{kg} / \mathrm{day}$ as free base) that has been previously shown to produce nicotinic receptors up-regulation, both in the brain and in blood cells. With such a low dose, nicotine withdrawal didn't affect locomotion nor anxiety levels but increased the number of rearing, jumping, and marginally, body-scratching. Other behaviours, classically considered to contribute to withdrawal syndrome, were unaffected, e.g., grooming, body or forelimb shakes. Our results show that anxiety may be dissociated from the behavioural withdrawal syndrome. Also, the severity of the syndrome produced by nicotine withdrawal is qualitatively and quantitatively different from the one induced by other drugs of
\end{abstract}

abuse and also by the one produced by nicotine at higher doses.

Keywords: Withdrawal; Anxiety; Locomotor Activity; Nicotinic Receptors

\section{INTRODUCTION}

Nicotine is known to be an addictive substance in humans as well as in other mammals [1-3]. As such, behavioral studies in animal models show that nicotine induces a withdrawal syndrome $[4,5]$, associated with the increased exhibition of multiple heterogeneous behaviors, many of which being shared by other drugs of abuse $[2,6]$. These findings, however, are confounded by two major factors: 1) in most cases, nicotine withdrawal is not associated with the same severity of symptoms as other drugs of abuse [6]; 2) global scores alone are not highly informative as they may reflect the grouping of different processes and different origins. Indeed, withdrawal signs are constituted of exaggerated levels of behaviours that are part of the normal behavioural repertoire of the animal, and as any behaviour, they may be influenced by multiple parameters such as genetic strain, individual and group level of stress, experimenter manipulation, lightning level or noise.

Authors of previous studies targeted several behaviours that are affected by nicotine as well as by other addictive drugs, i.e., rearing, abdominal constriction, grooming, scratching, chewing, cage scratching, and head nodding and jumping [7-10], and established a global abstinence score. In order to match with what others previously established, we scored the same behaviours but individually. Further, we scored the frequency of these behaviours in the same animals, before, during and after nicotine administration, in order to question whether their frequency varies within and between individuals and/or represents robust indicators of withdrawal. We therefore expected to provide evidence regarding which of these behaviours are specifically affected by nicotine 
withdrawal. We chose to administer continuously a low dose of nicotine $(2.4 \mathrm{mg} / \mathrm{kg} /$ day, as free-base $)$, via osmotic mini-pumps, as it was previously shown that it is sufficient to produce nicotinic receptor up-regulation in the mouse [11] and to induce withdrawal syndrome in the rat [12]. In accordance with previous studies $[7,8,10,12,13]$, we then used $1 \mathrm{mg} / \mathrm{kg}$ of mecamylamine to precipitate homogeneously the withdrawal the last day of mini-pump theoretical depletion.

Withdrawal from chronic use of nicotine also results in increased anxiety $[14,15]$, and reduced locomotor activity $[8,12,16-20]$, although these last processes were altered either after higher doses of nicotine than the one we chose, or in rats instead of mice. We have therefore tested the effects of nicotine withdrawal, after the low dose administered, on locomotor activity and anxiety behaviours of the mouse.

Experiments have been carried out following the guidelines of the European Communities Council (86/609/EEC). Twenty-two C57BL6/J male mice (3 to 5 months) supplied by Charles River Laboratories (France) were housed individually upon arrival under a $12 \mathrm{~h}$ light-dark cycle in rooms at a controlled temperature $\left(21^{\circ} \mathrm{C}\right)$. All experiments were carried out during the light phase, from 10 am to $6 \mathrm{pm}$. They were handled during approximately ten days before the beginning of the tests. They were slightly food deprived one week before the start of the experiments with their weight adjusted at $90 \%$ of their normal body weight, as the same animals were subsequently used in a learning experiment, in order to minimize the use of animals. However, in order to reduce anxiety potentially induced by food deprivation, animals were fed before the experiments.

After handling, mice were habituated 15 minutes/day for three days to the environment (a large $50 \mathrm{~cm} \mathrm{X} 30 \mathrm{~cm}$ transparent box, containing clean bedding) in which the behaviours were scored. Two experimenters, blind to the drug condition of the animals, scored the behaviours on line. Before mini-pump implantation, we first established the baseline level of each of the following behaviours: rearing, grooming, body shakes, body tremor and abdominal constriction, jumping, scratching, forelimb shake, chewing and head shakes. After the baseline measurement, mini-pumps were implanted subcutaneously under general anesthesia (a combination of xylazine, $2.5 \%$, and ketamine, $15 \%$ in $82.5 \%$ of PBS). Mini-pumps (Alzet) were filled with either nicotine $(2.4$ $\mathrm{mg} / \mathrm{kg} /$ day free base) or saline delivered at a constant rate $(0.25 \mu \mathrm{l} /$ hour $)$ for 28 days. In order to measure the effect of chronic nicotine administration, we scored the same behaviours a second time two weeks after mini-pump implantation. At the last day of mini-pump delivery, i.e., the $28^{\text {th }}$ day, mecamylamine was injected 15-20 minutes before the beginning of the withdrawal test at a dose of 1 $\mathrm{mg} / \mathrm{kg}$, IP. The same behaviours were then scored subsequently to evaluate the effects of nicotine withdrawal.
Locomotor activity was measured for 30 minutes in an empty large open field ( $1 \mathrm{~m}$ in diameter) made of white plastic. It was situated in a room containing distal visual cues with light level set between 80 and 100 Lux and was placed under a camera connected to a video-track system that allows the discrimination of navigatory -speed $>$ $11.8 \mathrm{~cm} / \mathrm{sec}$ - and exploratory movements -speed < $6.8 \mathrm{~cm} / \mathrm{sec}-[17]$. It has been shown that rodents spontaneously avoid the center of a large open area which is more brightly illuminated than the periphery [21]. We therefore used the number of visits and time spent in the center as an index of anxiety during free exploratory activity. The baseline level of anxiety is altered by novelty [22]. Therefore, in order to avoid the alteration of the anxiety level generated by the first visit of a new environment, we conducted exploratory experiments in distinct groups of mice under the chronic administration of nicotine or saline and during withdrawal of nicotine or saline.

Each behavior was analyzed using repeated measures analysis of variance (ANOVAs) with "Drug condition" as the main within-subject factor, with 3 levels (before implantation, under chronic treatment and withdrawal), and "treatment" (with 2 levels, saline and nicotine) as the main between-subjects factors. Upon significant main effect (i.e., for $\mathrm{p}<0.05$ ), data was further analyzed with paired t-tests.

Statistical analysis revealed a significant effect of the main within-subject factor, drug condition, for rearing $\left(\mathrm{F}_{(2,40)}=15.38, \mathrm{p}<0.0001\right)$, jumping $\left(\mathrm{F}_{(2,40)}=3.98, \mathrm{p}<0.026\right)$, body-scratching $\left(\mathrm{F}_{(2,40)}=5.05, \mathrm{p}=0.01\right)$, grooming $\left(\mathrm{F}_{(2,40)}=\right.$ $3.23, \mathrm{p}=0.05)$ and forelimb shakes $\left(\mathrm{F}_{(2,40)}=12.23, \mathrm{p}<\right.$ $0.0001)$, suggesting that, individually, each of these parameters were altered either by the treatment and/or the repetition of the test. We also observed a significant interaction drug condition $\mathrm{X}$ treatment for rearing $\left(\mathrm{F}_{(2,40)}\right.$ $=3.96, \mathrm{p}=0.027)$, suggesting that nicotine and saline treatment produced a different effect on rearing. By contrast, no significant effect of drug condition and no interaction drug condition $\mathrm{x}$ treatment were observed for abdominal constriction (respectively, $\mathrm{F}<1$ and $\mathrm{F}_{(2,40)}=1.61$, NS) suggesting that this variable was not altered by the drug condition and, therefore, may not contribute significantly to the withdrawal syndrome.

When measured under chronic treatment, rearing $(\mathrm{t}=-0.86, \mathrm{p}=0.4, \mathrm{df}=20)$ and jumping $(\mathrm{t}=0.34, \mathrm{p}=0.74$, $\mathrm{df}=20$ ) levels were similar in animals receiving saline and those receiving nicotine, making both variables unaffected by the chronic administration of nicotine, as illustrated in Figure 1. Grooming was the only behaviour that showed a significant main effect of group $\left(F_{(1,20)}=7.44\right.$, $\mathrm{p}=0.013)$ and a significant main effect of drug condition $\left(\mathrm{F}_{(2,40)}=3.23, \mathrm{p}=0.05\right)$ with no significant interaction $(\mathrm{F}<1$, NS), suggesting that the drug condition was identical for the group receiving saline and the one receiving nicotine. It further suggests that the group effect (i.e. nicotine effect) was not dependent of the drug condition, i.e., both groups 


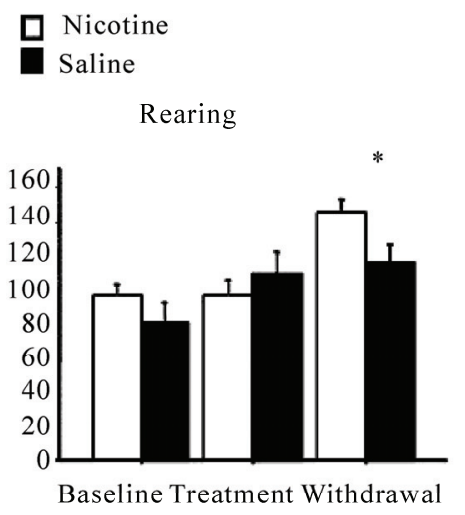

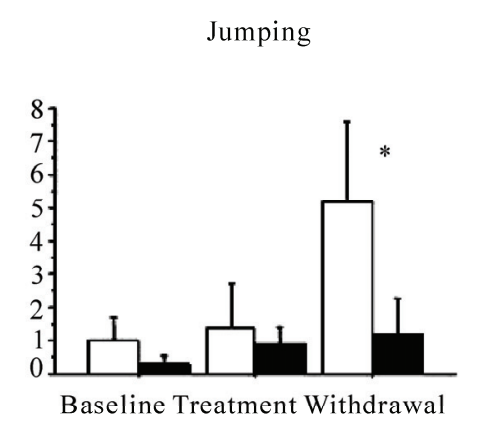

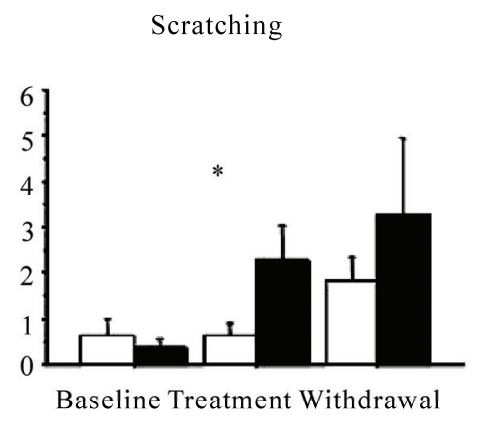

Grooming

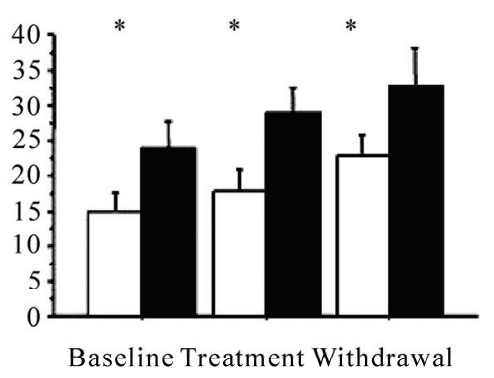

Forelimb Shakes

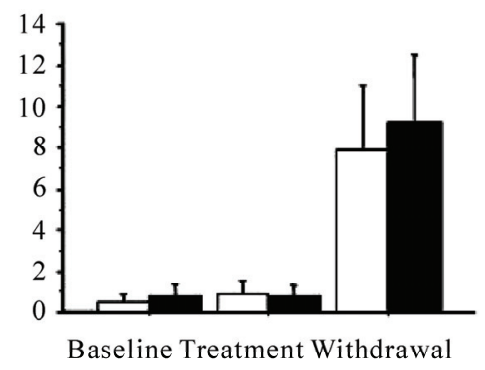

(Fig. 1)

Figure 1. Quantification of spontaneous behaviours affected by chronic administration of nicotine and nicotine withdrawal precipitated by injection of mecamylamine. Rearing, jumping, scratching, grooming and forelimb shakes were scored before mini-pump implantation (baseline), under chronic nicotine (white) or saline (black) treatment, and the last day of mini-pump delivery, $20 \mathrm{~min}$ after mecamylamine injection (withdrawal). $*$ indicates a significant difference between animals under saline and those under nicotine.

may show different levels of grooming before mini-pump implantation. Post-hoc tests confirmed that the level of grooming was marginally different between the saline and the nicotine groups at baseline condition $(\mathrm{t}=-1.95$, $\mathrm{p}=0.065, \mathrm{df}=20$ ) and was significantly different under chronic treatment $(\mathrm{t}=-2.38, \mathrm{p}=0.027, \mathrm{df}=20)$. These results suggest that nicotine had no effect by itself on grooming but that both groups, analysed by experimenters blind to the drug condition of the animals, were different regarding this parameter from the beginning of the experiment.

Body-scratching was marginally affected by the chronic drug treatment $(\mathrm{t}=-2.05, \mathrm{p}=0.054, \mathrm{df}=20)$. As illustrated in Figure 1, and confirmed by paired t-tests, animals receiving saline showed significantly less body- scratching levels when tested on baseline than when tested under mini-pumps $(\mathrm{p}=0.026)$ whereas animals receiving nicotine did not show different level of body- scratching between the two testing conditions $(p=1.0)$. This suggests that the repetition of the test affected only animals that were not under nicotine treatment.

Forelimb shakes were unaffected by nicotine administration as the number of paw tremor recorded was not significantly different between animals receiving saline and those receiving nicotine under chronic treatment $(\mathrm{t}=0.17, \mathrm{p}=0.87, \mathrm{df}=20)$.

Post-hoc tests confirmed that the levels of rearing $(\mathrm{t}=-7.03, \mathrm{p}<0.0001, \mathrm{df}=10)$, jumping $(\mathrm{t}=-2.33, \mathrm{p}=0.042$, $\mathrm{df}=10)$, body-scratching $(\mathrm{t}=-2.14, \mathrm{p}=0.058, \mathrm{df}=10)$ and forelimb shakes $(\mathrm{t}=-2.58, \mathrm{p}=0.027, \mathrm{df}=10)$ were significantly different under nicotine administration and during withdrawal. The levels of rearing, jumping and body scratching didn't change from chronic treatment to withdrawal condition in saline animals (respectively: $\mathrm{t}=0.4$, $\mathrm{p}=0.7, \mathrm{NS} ; \mathrm{t}=0.25, \mathrm{p}=0.81$, NS and $\mathrm{t}=-0.99, \mathrm{p}=0.35$, NS), suggesting that these behaviours were altered specifically by nicotine withdrawal, as illustrated in Figure 1 Forelimb shakes were significantly altered in mice under chronic administration of saline (paired t-test conducted for chronic condition versus withdrawal condition, $\mathrm{t}=-2.7$, $\mathrm{p}=0.022, \mathrm{df}=10$ ), suggesting that this behaviour was affected by mecamylamine administration but not by nicotine withdrawal. Post-hoc tests showed that nicotine withdrawal did not affect grooming $(\mathrm{t}=-1.43, \mathrm{p}=0.18$, $\mathrm{df}=10$ ). 

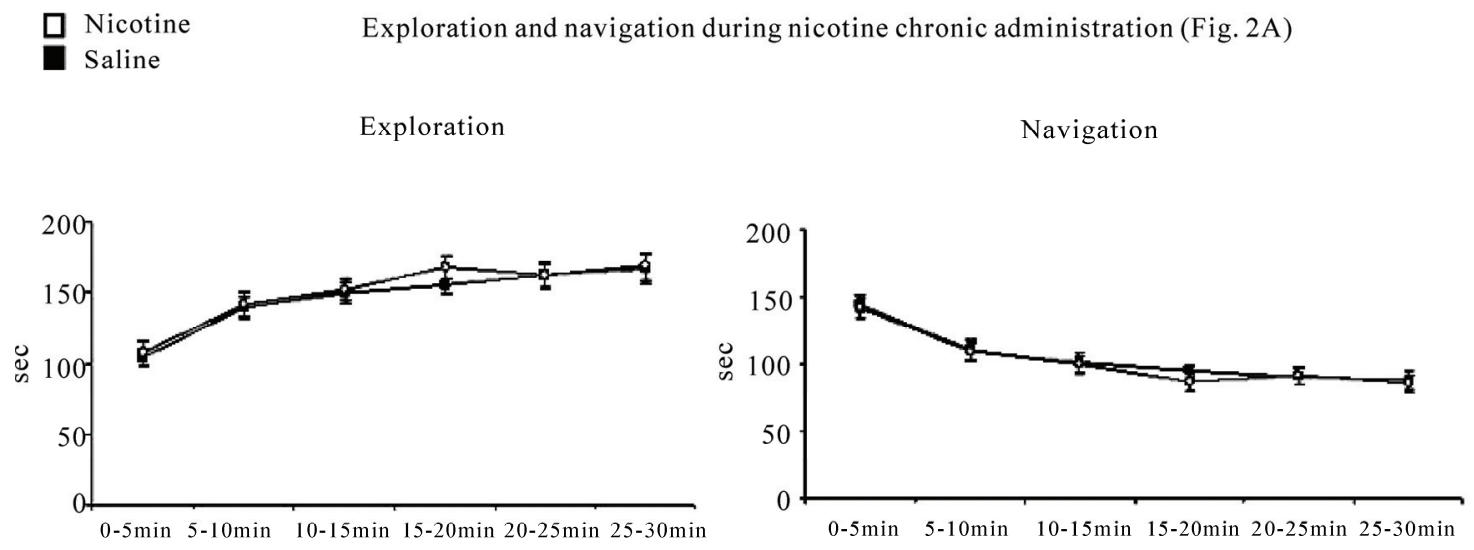

Exploration and navigation after nicotine withdrawal (Fig. 2B)

Exploration

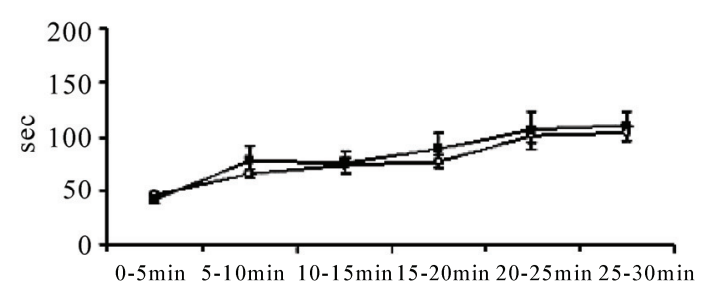

Navigation

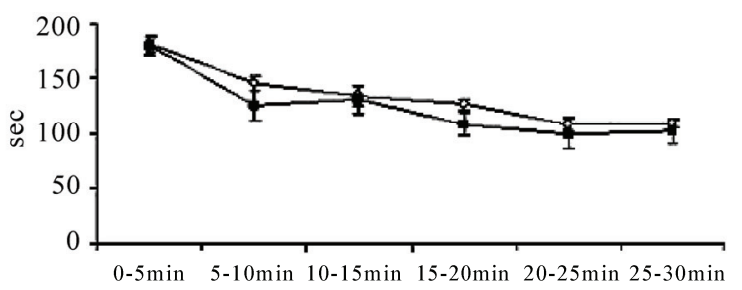

Figure 2. Exploratory and navigatory activity recorded in the circular open field for $30 \mathrm{~min}$ during chronic treatment (A) and during withdrawal (B). No significant difference was found between animals under saline (black) and those under nicotine (white).

Navigation and exploration were not affected by the chronic administration of nicotine, as illustrated in Figure $2 \mathrm{~A}$ ANOVAs confirmed that there was no treatment effect on navigation nor on exploration (both $\mathrm{Fs}<1$, NS). For both types of behaviour, there was a significant effect of habituation (for navigation: $\mathrm{F}_{(5,85)}=44.6, \mathrm{p}<0.0001$; for exploration: $\left.F_{(5,85)}=31.85, p<0.0001\right)$ and no significant interaction treatment $\mathrm{X}$ habituation (both $\mathrm{Fs}<1$, NS) suggesting that subjects receiving saline as well as those receiving nicotine showed normal habituation.

Navigation and exploration (Figure 2) were not statistically different after withdrawal in saline and in nicotine animals, as there was no treatment effect neither for navigation $\left(\mathrm{F}_{(1,12)}=1.05\right.$, NS) nor for exploration $(\mathrm{F}<1$, NS). Within 30 minutes, there was a significant habituation for both navigation $\left(\mathrm{F}_{(5,60)}=57.96, \mathrm{p}<0.0001\right)$ and exploration $\left(\mathrm{F}_{(5,60)}=25.02, \mathrm{p}<0.0001\right)$ but no significant interaction treatment $\mathrm{X}$ habituation for either navigation $\left(\mathrm{F}_{(5,60)}=1.1, \mathrm{p}=0.37, \mathrm{NS}\right)$ or exploration $(\mathrm{F}<1, \mathrm{NS})$. These results showed that the levels of exploratory and navigatory activity as well as habituation were not affected by nicotine withdrawal.

The chronic administration of nicotine didn't affect the number of entries in the centre (data not shown, ANOVAs for drug effect: $F_{(1,17)}=2.45$, NS) but produced a trend to reduce the time spent in the centre of the arena (Figure 3A, ANOVAs for drug effect: $\mathrm{F}_{(1,17)}=4.33, \mathrm{p}=0.053$ ), suggesting that, in an unfamiliar environment, chronic nicotine administration tends to increase anxiety level.

The total number of entries in the centre (data not shown) and the time spent in the centre of the open field in 30 minutes (Figure 3B) were not different after withdrawal between saline and nicotine animals, as confirmed by ANOVAs (no drug effect for number of entries: $\mathrm{F}<1$, NS; no drug effect for time in the centre: $\mathrm{F}<1$, NS). These results showed that the level of anxiety was not affected by nicotine withdrawal in an unfamiliar environment.

Our present results demonstrate that only rearing, jumping and body-scratching reflect specifically nicotine withdrawal syndrome in mice, when nicotine dependence is produced by the continuous administration of a low dose i.e., $2.4 \mathrm{mg} / \mathrm{kg} /$ day. This dose has been shown to induce a significant up-regulation of nicotinic receptors both in the blood and in the brain [11]. We find that in these conditions nicotine withdrawal affects neither anxiety nor locomotion. Previous studies have used significantly higher doses, i.e., up to ten times higher, that induced withdrawal signs of different nature than the one we observed in the present study but that also increased anxiety level $[9,14,15]$. Interestingly, when using a dose 
$\square$ Nicotine

Anxiety level during nicotine administration (Fig. 3A)

Saline

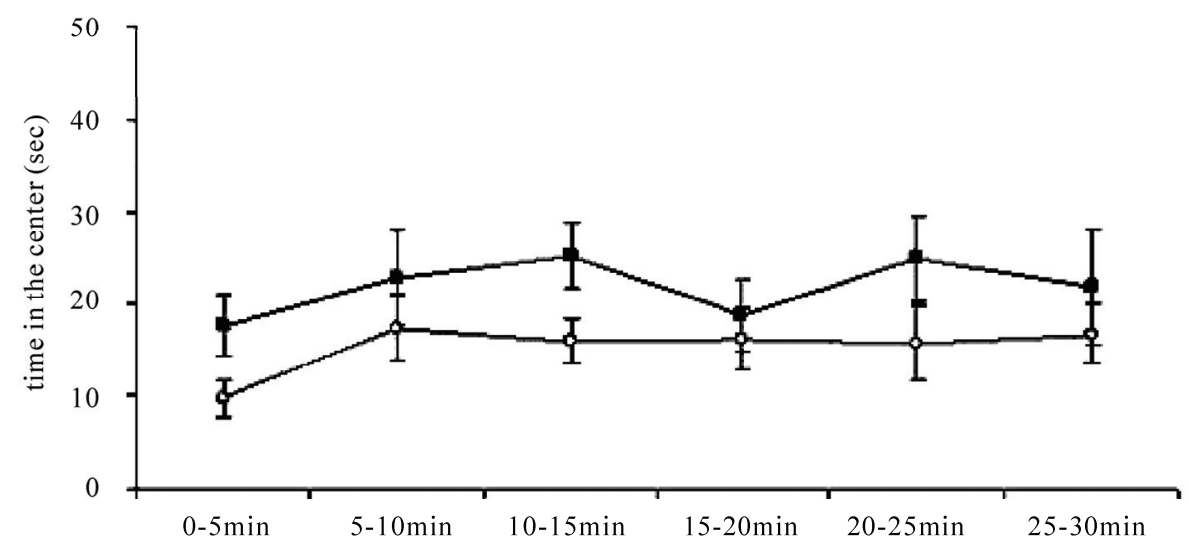

Anxiety level after nicotine withdrawal (Fig. 3B)

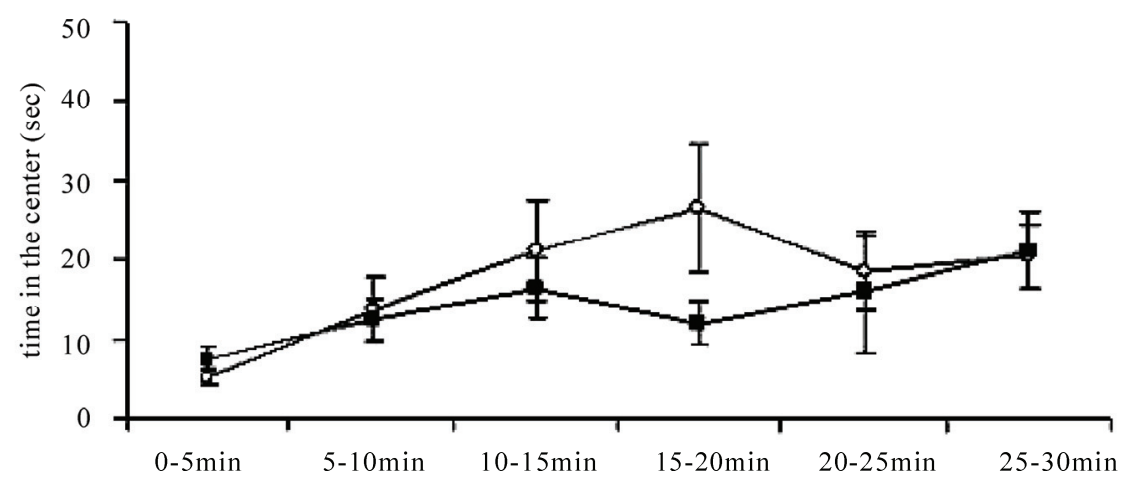

Figure 3. Time spent in the centre of the brightly illuminated open field over one single 30-minute session during chronic treatment (A) and during nicotine withdrawal (B). Under chronic nicotine, mice tended to decrease the time spent in the centre of the open field, as compared to animals receiving saline (A). No significant difference was found between animals receiving saline (black) and those receiving nicotine (white) during withdrawal (B).

in a range similar to our, but excluding rearing and jumping from their scores, Damaj and collaborators [14] did not find any withdrawal signs or anxiety alteration. Therefore, in agreement with previous studies using a large range of nicotine doses, our present results provide evidence that a low dose of nicotine given continuously also induces an increase in the frequency of some specific behaviour. Using a low dose of nicotine, we were able to show dissociation between anxiety and other behavioural processes that may influence the general withdrawal syndrome. This dissociation suggests that withdrawal signs may not be exclusively due to an increase in anxiety levels after nicotine abstinence. It must be noticed that our animals were under a slight food deprivation schedule and, although they were fed before behavioural recording, we cannot exclude that chronic food deprivation has modified the effects of nicotine withdrawal. We conducted experiments (unpublished results) showing that a slight chronic food deprivation doesn't alter by itself navigatory and exploratory processes. These experiments do not provide evidence that food deprivation does not interfere with the nicotine exposure, for example by increasing anxiety levels. Such data are not yet available, to our knowledge.

Rearing behaviours have been shown to be sensitive to manipulations of the dopaminergic system [23]. Hildebrand and collaborators [13] also showed in nicotinedependent rats that intra-Ventral Tegmental Area injec- 
tion of mecamylamine induced a rearing frequency modification with a dose of nicotine similar to our dose range. Therefore, it is possible that chronic nicotine administration followed by precipitated withdrawal created a deficit in cholinergic transmission which, in turn, reduced dopamine (DA) release, leading to an increased level of rearing. Microdialysis studies indeed showed that in nicotine-dependent rats, precipitated withdrawal leads to a decrease in DA release in the nucleus accumbency and in the prefrontal cortex $[24,25]$. Considering the role of the DA system in addiction [1], it is therefore not surprising that behaviours that are influenced by its modulation are particularly affected during withdrawal. Forelimb shakes are generally considered as a behavioural manifestation associated with drug withdrawal $[8,26]$. Our results showed that their frequency was affected by mecamylamine administration but not by nicotine withdrawal, as it was observable also in mice that were administered saline chronically. Previous studies [14,27] showed that this measure as well as some other signs are affected by hexamethonium or chlorisondamine, nicotinic antagonists which penetrate poorly the blood-brain barrier. Therefore, our data, along with previous ones [19] suggests that forelimb shakes are from peripheral origin and cannot be considered as a centrally-mediated withdrawal sign. We further show here that some of the behaviours usually recorded as part of a withdrawal syndrome, i.e., grooming, may be affected by the repetition of testing, but not by the nicotine treatment or withdrawal. It is furthermore noticeable that a baseline difference between groups (Figure 1), uncorrected as the experimenters were blind to the animals' treatment, remains observable for weeks, despite the habituation of the animals. This suggests that grooming is a behaviour that varies strongly between individuals, reinforcing the interest of longitudinal studies that allow baseline measurements. We also showed that body scratching was affected by nicotine administration and, only in animals that received nicotine, by withdrawal. Although this effect is statistically evidenced, it must be noticed that in absolute numbers, it is not highly represented in our data and should therefore be confirmed, despite the fact that it is classically associated with drug withdrawal [8,10,20,27]. Together with grooming data, scratching data emphasized the necessity to give baseline levels of such spontaneous behaviours when scoring drug effects.

In the open field, our data showed that mice under chronic administration of nicotine tend to spend less time in the centre of the arena, suggesting that, in an unfamiliar environment, chronic nicotine administration tends to increase anxiety. Our results also show that nicotine withdrawal, at a low dose, had no effect on anxiety when the anxiogenic environment was not familiar. This result is compatible with the one of other authors who compared the withdrawal effect after different doses of nicotine on anxiety [14].
Locomotor activity was affected neither by chronic nicotine exposure, nor by withdrawal. It is noticeable that, in rats, a similar dose of nicotine induced a temporary decrease of locomotor activity that was observable between 16 to 24 hours after mini-pump removal [6,12], but not in mice after pharmacological precipitation [28]. It is not possible to conclude firmly from experimental data obtained in different species (rats versus mice) and with two different procedures (mini-pump removal versus precipitation). Indeed, it is known that the sensitivity to nicotine is different in rats and mice [29]. Also, it is possible that mini-pump removal, that allows enough time for producing alteration in striatal DA concentration [12], produces a higher impact on locomotor activity than pharmacological precipitation, via DA mechanisms.

In conclusion, our present results support the idea that a continuous administration of low dose of nicotine, followed by pharmacological precipitation of withdrawal, is sufficient to produce a withdrawal syndrome in the mouse represented by increased levels of rearing and jumping. With such a low dose, nicotine withdrawal didn't affect locomotion nor anxiety levels. Other behaviours, contributing to nicotine withdrawal after higher doses of nicotine, were unaffected. Therefore, in spite of the fact that anxiety may produce some of the behaviours associated with nicotine withdrawal, other behaviours, unrelated with anxiety can also be affected. These results can be discussed in terms of a relatively dissociable role of nicotinic receptors in the reward versus somatic dependence systems.

The future determination of whether these behaviours rely on specific neurotransmitter systems or brain structures is expected to complement the current therapeutical proposal of the withdrawal syndrome [30, for review].

\section{REFERENCES}

[1] Chiara, Di G. (2000) Role of dopamine in the behavioural actions of nicotine related to addiction. Eur. J. Pharmacol, 393, 295-314.

[2] Epping-Jordan, M.P., Watkins, S.S., Koob, G.F. and Markou, A. (1998) Dramatic decrease in brain reward function during nicotine withdrawal. Nature, 393, 76-79.

[3] Rose, J.E. and Corrigall, W.A. (1997) Nicotine self-administration in animals and humans: Similarities and differences. Psychopharmacology, 130, 28-40.

[4] Aston-Jones, G. and Harris, G.C. (2004) Brain substrates for increased drug seeking during protracted withdrawal. Neuropharmacol, 47, 167-179.

[5] Stolerman, I.P. and Jarvis, M.J. (1995) The scientific case that nicotine is addictive. Psychopharmacology, 117, 2-10.

[6] Malin, D.H., Lake, J.R., Newlin-Maultsby, P., Roberts, L.K., Lanier, J.G., Carter, V.A., Cunningham, J.S. and Wilson, O.B. (1992) Rodent model of nicotine abstinence syndrome. Pharmacol. Biochem. Behav., 43, 779-784.

[7] Castané, A., Valjent, E., Ledent, C., Parmentier, M., 
Maldonado, R. and Valverde, O. (2002) Lack of CB1 cannabinoid receptors modifies nicotine behavioural responses, but not nicotine abstinence. Neuropharmacology, 43, 857-867.

[8] Isola, R., Vogelsberg, V., Wemlinger, T.A., Neff, N.H. and Hadjiconstantinou, M. (1999) Nicotine abstinence in the mouse. Br. Res., 850, 189-196.

[9] Salas, R., Pieri, F., and Biasi, De M. (2004) Decreased signs of nicotine withdrawal in mice null for the 4 nicotinic acetylcholine receptor subunit. J. Neurosci., 24, 10035-10039.

[10] Salmon, A.M., Evrard, A., Damaj, I. and Changeux, J.P. (2004) Reduction of withdrawal signs after chronic nicotine exposure of alpha-calcitonin gene-related peptide knock-out mice. Neurosci Lett., 360, 73-76.

[11] Cormier, A., Paas, Y., Zini, R., Tillement, J.P., Lagrue, G., Changeux, J.P. and Grailhe, R. (2004) Long-term exposure to nicotine modulates the level and activity of acetylcholine receptors in white blood cells of smokers and model mice. Molec. Pharmacol, 66, 1712-1718.

[12] Fung, Y.K., Schmid, M.J., Anderson, T.M. and Lau, Y.S. (1996) Effects of nicotine withdrawal on central dopaminergic systems. Pharmacol. Biohem. Behav, 53, 635- 640.

[13] Hildebrand, B.E., Nomikos, G.G., Bondjers, C., Nisell, M. and Svensson, T.H. (1997) Behavioural manifestations of the nicotine abstinence syndrome in the rat: Peripheral versus central mechanisms. Psychopharmacology, 129, 348-356.

[14] Damaj, M.I., Kao, W. and Martin, B.R. (2003) Characterization of spontaneous and precipitated nicotine withdrawal in the mouse. J. Pharmacol. Exp. Ther. 307, 526-534.

[15] Jonkman, S., Henry, B., Semenova, S. and Markou, A. (2005) Mild anxiogenic effects of nicotine withdrawal in mice. Eur. J. Pharmacol, 516, 40-45.

[16] Gäddnäs, H., Pietilä, K. and Ahtee, L. (2000) Effects of chronic oral nicotine treatment and its withdrawal on locomotor activity and brain monoamines in mice. Behav. Br. Res, 113, 65-72.

[17] Granon, S., Faure, P. and Changeux, J.P (2003) Executive and social behaviours under nicotinic receptor regulation. Proc Natl Acad Sci USA, 100, 9596-9601.

[18] Helton, D.R., Modlin, D.L., Tizzano, J.P. and Rasmussen, K. (1993) Nicotine withdrawal: A behavioural assessment using schedule controlled responding, locomotor activity, and sensorimotor reactivity. Psychopharmacology, 113, 205-210.
[19] Hildebrand, B.E., Panagis, G., Svensson, T.H. and Nomikos, G.G. (1999) Behavioural and biochemical manifestations of mecamylamine-precipitated nicotine withdrawal in the rat: Role of nicotinic receptors in the ventral tegmental area. Neuropsychopharmacology, 21, 560-574.

[20] Malin, D.H., Lake, J.R., Carter, V.A., Cunningham, J.S., Hebert, K.M., Conrad, D.L. and Wilson, O.B. (1994) The nicotine antagonist mecamylamine precipitates nicotine abstinence syndrome in the rat. Psychopharmacology, 115, 180-184.

[21] Lipkind, D., Sakov, A., Kafkafi, N., Elmer, G.I., Benjamini, Y. and Golani, I. (2004) New replicable anxiety-related measures of wall vs centre behaviour of mice in the open field. J. Appl. Physiol., 97, 347-59.

[22] Crawley, J.N. (2000) What's wrong with my mouse? Behavioural phenotyping of transgenic and knockout mice. John Wiley-Liss Publication.

[23] Fujita, S., Adachi, K., Lee, J., Uchida, T., Koshikawa, N. and Cools, A.R. (2004) Decreased postsynaptic dopaminergic and cholinergic functions in the ventrolateral striatum of spontaneously hypertensive rats. Eur. $J$. Pharmacol, 484, 75-82.

[24] Carboni, E., Bortone, L., Giua, C. and Chiara, Di G. (2000) Dissociation of physical abstinence signs from changes in extra cellular dopamine in the nucleus accumbens and in the prefrontal cortex of nicotine dependent rats. Drug Alcohol Depend, 58, 93-102.

[25] Rada, P., Jensen, K. and Hoebel, B.G. (2001) Effects of nicotine and mecamylamine-induced withdrawal on extracellular dopmaine and acetylcholine in the rat nucleus accumbens. Psychopharmacology, 157, 105-110.

[26] Maldonado, R. (2002) Study of cannabinoid dependence in animals. Pharm. Ther, 92, 153-164.

[27] Kenny, P.J. and Markou, A. (2001) Neurobiology of the nicotine withdrawal syndrome. Pharmacol. Biochem. Behav., 70, 531-549.

[28] Balerio, G.N., Aso, E., Berrendero, F., Murta, P. and Maldonado, R. (2004) D9-Tetrahydrocannabinol decreases somatic and motivations of nicotine withdrawal in mice. Eur. J. Neurosci, 20, 2737-2748.

[29] Spielewoy, C. and Markou, A. (2004) Strain-specificity in nicotine attenuation of phencyclidine-induced disruption of prepulse inhibition in mice: Relevance to smoking in schizophrenia patients. Behav. Genet., 34, 343-354.

[30] Berrettini, W.H. and Lerman, C.E. (2005) Pharmacotherapy and pharmacogenetics of nicotine dependence. Am. J. Psychiatry, 162, 1441-1451. 